\title{
As Good as New. How to Successfully Recycle English GPT-2 to Make Models for Other Languages
}

\author{
Wietse de Vries \\ University of Groningen \\ The Netherlands \\ wietse.de.vries@rug.nl
}

\author{
Malvina Nissim \\ University of Groningen \\ The Netherlands \\ m.nissimerug.nl
}

\begin{abstract}
Large generative language models have been very successful for English, but other languages lag behind, in part due to data and computational limitations. We propose a method that may overcome these problems by adapting existing pre-trained models to new languages. Specifically, we describe the adaptation of English GPT-2 to Italian and Dutch by retraining lexical embeddings without tuning the Transformer layers. As a result, we obtain lexical embeddings for Italian and Dutch that are aligned with the original English lexical embeddings. Additionally, we scale up complexity by transforming relearned lexical embeddings of GPT- 2 small to the GPT- 2 medium embedding space. This method minimises the amount of training and prevents losing information during adaptation that was learned by GPT-2. English GPT-2 models with relearned lexical embeddings can generate realistic sentences in Italian and Dutch. Though on average these sentences are still identifiable as artificial by humans, they are assessed on par with sentences generated by a GPT-2 model fully trained from scratch.
\end{abstract}

\section{Introduction}

Large pre-trained language models have brought unprecedented progress in NLP, but also concerns regarding the excessive computing power needed to train them (Strubell et al., 2019). Limited access to large amounts of computational resources, as well as environmental considerations, curb possibilities for less-resourced and less-researched languages. Additionally, models like GPT-2 (Radford et al., 2019) are trained on amounts of data that are not available for most languages. As a result of these limitations, language models are commonly trained for English, whereas reproductions in other languages may underperform or not exist.

That language models can benefit from information in other languages has been demonstrated by the effectiveness of multilingual BERT (mBERT) and XLM-RoBERTa (Conneau et al., 2020). However, for downstream tasks mBERT has been shown to be outperformed by monolingual models for higher resource languages whereas lower resource languages can still achieve better results without pre-trained language models (Nozza et al., 2020; Wu and Dredze, 2020).

Rather than pursuing a multilingual direction, we aim at exploiting existing language models and language similarities to create models for new languages. Specifically, we develop a multi-step procedure for adapting English GPT-2 (Radford et al., 2019) to Italian and Dutch. Dutch is genetically closely related to English, both being WestGermanic languages, while Italian is a more distant Romance language from the same Indo-European language family (Eberhard et al., 2020). It is however worth noticing that at sentence level English and Italian tend to have the same word order (SVO), while Dutch is SVO in main clauses, but SOV in subordinate ones; at noun phrase level, English and Dutch share constituent order (for example adjective-noun) while Italian is different (mostly noun-adjective). A GPT-2 based model has previously been trained from scratch for Italian (De Mattei et al., 2020). We can thus compare sentences generated by this model with sentences generated by our adapted model. For Dutch, no other GPT-2 based models exist, but similar BERT-based models have been trained from scratch (de Vries et al., 2019; Delobelle et al., 2020).

Procedure Overview and Contributions When training a new language model, weights of an existing pre-trained model for another language can be used for initialisation. The first step in our training procedure is to only retrain the lexical embeddings of the GPT-2 small model, without touching the Transformer layers. We show that retrained lexical embeddings are well aligned with the English vo- 
cabulary and that GPT-2 is capable of generating realistic text in Italian and Dutch after this step. Next, we demonstrate that the lexical embeddings of larger GPT-2 models can be approximated by transforming the small lexical embeddings to the GPT-2 medium lexical embedding space. The leastsquares regression method is the most effective transformation method for this scaling procedure. Human judgements show that generated sentences are often realistic, but become even more consistently so after additional finetuning of the Transformer layers. This improvement is stronger for Dutch than for Italian.

The steps in our pipeline yield GPT-2 based language models for Italian and Dutch which are made available on the Hugging Face model hub ${ }^{1}$; the source code is available on Github ${ }^{2}$. On the last page, we also include a 'recipe' for creating GPT-2 models for new languages.

\section{Background}

Previous and current research relevant for the present work is found in the more general field of transfer learning, with a specific focus on language transfer. We also discuss how our approach of translating lexical layers in different model sizes relates to work on aligning word embeddings.

\subsection{Language transfer}

Transfer learning can be an effective strategy to adapt models to lower-resource languages by initially training a model for a source language and then further training (parts of) the model for a target language. It has been successfully used to create machine translation models with little parallel data (Zoph et al., 2016) as well as other classic NLP tasks (Lin et al., 2019).

In machine translation a model can be adapted by initially training it for a high-resource language pair after which the model should be partially retrained for a low-resource language (Zoph et al., 2016; Nguyen and Chiang, 2017; Kocmi and Bojar, 2018). Retraining a randomly initialised lexical layer while freezing the rest of the model is an effective method to adapt a model to a new language, and dictionary based initialisation is not required to get the best performance (Zoph et al., 2016). Artetxe et al. (2020) show that a monolin-

\footnotetext{
${ }^{1}$ https://huggingface.co/GroNLP

${ }^{2}$ https://github.com/wietsedv/ gpt2-recycle
}

gual BERT model can be adapted from a source language to a different target language by retraining the lexical layer for the target language while freezing the Transformer layers in the model. Zero shot adaptation for downstream tasks is possible by finetuning the original source model with source language data and swapping lexical layers afterwards. Lexical layer retraining approaches may be effective despite the presence of source and target language dissimilarities if a downstream task does not require perfect data. However, these methods have not been applied yet to generative language models where dissimilarities can cause clear syntactic and lexical errors.

Language similarity plays a role in the effectiveness of transfer learning for language models. For instance, in machine translation French is a better parent model for Spanish than German (Zoph et al., 2016). Word order differences between languages can negatively influence transfer performance, and Kim et al. (2019) show that randomly swapping words in the source language, which forces the model to rely less on consistent word order, can improve performance in the target language. Overall, genetic similarity between source and target languages can play a role, but Lin et al. (2019) have shown that in practice the geographic distances between countries of origin, syntactic similarity and subword overlap are better predictors of transfer performance for machine learning, part-of-speech tagging, dependency parsing and entity linking.

\subsection{Aligning word embeddings}

Alignment of lexical embeddings, for example for multiple languages, is most prominently done with mapping-based approaches (Ruder et al., 2019). Typically, a function is determined that transforms one vector space to another based on a seed lexicon. This lexicon is a dictionary of anchor points that should result close together after transformation.

An influential method for learning a lexical embedding mapping is the least-squares linear transformation method by Mikolov et al. (2013). They observe that words and their translations in other languages show similar constellations of related words after such a transformation. An alternative method that is generally considered to be an improvement (Ruder et al., 2019) is the orthogonal procrustes solution. This method adds the constraint that the transformation matrix must be orthogonal. In practice this means that the transfor- 
mation only contains rotations and reflections and no scaling and translation. This constraint enables length normalisation (Xing et al., 2015) and ensures monolingual invariance (Artetxe et al., 2016).

Mapping-based approaches rely on isomorphism, which means that a one-to-one token mapping between source and target lexical embedding spaces should be possible. This assumption is used for bilingual lexicon induction after alignment (Conneau et al., 2018). However, the isomorphism assumption highly depends on language similarity and (amount of) training data (Søgaard et al., 2018). Some more complex alignment methods like RCLS (Joulin et al., 2018) optimise for dictionary translation performance, which assumes isomorphism, but simpler methods like the orthogonal procrustes solution are more effective for downstream tasks like natural language inference (Glavaš et al., 2019). Mohiuddin et al. (2020) propose a solution to the isomorphism problem by learning a new shared embedding space with an auto-encoding neural model instead of trying to fit the embeddings of one language in the space of another language.

\section{Resources}

Models The models that we train are based on the pre-trained GPT-2 language models (Radford et al., 2019). GPT-2 is an auto-regressive Transformerdecoder based language model for English and comes in four sizes: small (12 layers), medium (24 layers), large (36 layers) and extra large (48 layers). Our experiments use the small (sml) and medium (med) model sizes.

Pre-training data The GPT-2 models are (further) pre-trained with Italian (ita) and Dutch (nld) data. The Italian pre-training data is the same dataset that was used to train the Italian GPT-2 small language model GepPpeTto (De Mattei et al., 2020). This dataset is a combination of Wikipedia data (2.8GB) and web texts from the ItWaC corpus (11GB; Baroni et al. 2009). Dutch data consists of a combination of Wikipedia (2.0GB), newspaper articles (2.9GB; Ordelman, Roeland J.F. et al. 2007), books (6.5GB) and articles from various Dutch news websites (2.1GB). Documents are filtered to only contain Dutch texts using the Wikipedia-trained fastText language identifier (Joulin et al., 2017), and are deduplicated based on exact sentence matches. The final Dutch pre-training data contains $13 \mathrm{~GB}$ of plain text, of which $5 \%$ is reserved as development data.
Evaluation data The Italian models are tested using the same three corpora that were used to evaluate GePpeTto (De Mattei et al., 2020): Wikipedia, ItWaC, EUR-Lex (laws), newspapers and blog posts. A 5\% subset of this data is used for development. For perplexity evaluation, the Dutch 500 million word, 22-genre SoNaR corpus is used (Oostdijk et al., 2013). The smaller 1 million word SoNaR-1 subcorpus is used as development data.

Tokenisation The datasets are tokenised using byte-pair-encoding (BPE). For better comparison, the Italian vocabulary is taken from the GePpeTto model (De Mattei et al., 2020). The Dutch BPE vocabulary is based on the full pre-training data and it has been ensured that every character that is used in the Dutch language is present as a single character token in the vocabulary. A large vocabulary size is beneficial because words are less often split in separate tokens, but vocabularies that are too large will have low token coverage for uncommon tokens.

Computation Training a model like GPT- 2 is a computationally expensive task that requires access to costly hardware for long training times. All models discussed in this paper are trained with eight parallel NVIDIA V100 32GB GPUs on the Peregrine high performance computing cluster at the University of Groningen. For efficient implementation of the models, we use PyTorch (1.6.0; Paszke et al. 2019), PyTorch Lightning (0.9.0; Falcon 2019) and Transformers (3.0.2; Wolf et al. 2020). We implement four strategies to decrease general training time. First, the models are trained with 16-bit automatic mixed-precision training (Micikevicius et al., 2018). This decreases training time with a factor of two to three times. Second, we split each document in windows of 128 instead of 1024 tokens when we only train the lexical embeddings. Third, we minimise padding by using bucketed random sampling which means that sequences within minibatches have roughly the same length. Finally, we use maximum batch sizes that fit into GPU memory and use gradient accumulation in order to do backpropagation only for every 2000 examples. The models are trained with the Adam optimiser (Kingma and Ba, 2017) and initial learning rates are chosen based on the steepest loss slope with gradually increasing learning rates (Smith, 2017). The learning rate is reduced by $10 \%$ on when training loss reaches a plateau. More implementation details are given in the git repository. 


\section{Cross-language Transfer}

We adapt GPT-2 for Italian and Dutch with minimal random initialisation. The lexical embeddings in GPT-2 are trained with an English BPE vocabulary. Therefore, they are not usable for the new languages and the lexical embedding layer has to be randomly initialised for the target vocabulary. This lexical embedding layer is used both as the first and the last layer of GPT-2 (tied weights). Relearning lexical embeddings with frozen Transformer layers prevents catastrophic forgetting in the Transformer layers when the embeddings are still random.

Relearning lexical embeddings Relearning lexical embeddings is nearly as computationally expensive as fully training the model, because backpropagation has to be done through the full model in order to update the lexical embeddings in the first layer of the model. However, loss values stabilize after only one to two epochs with lexical embedding relearning whereas full model training takes more training time. We retrain the lexical embeddings for the sml and med model for Italian and Dutch by training until loss on the validation data stops decreasing. When we retrain the sml model, the perplexities on our Italian and Dutch test data become 44.2 and 48.9 respectively. These perplexity scores show that the sml model can predict Dutch and Italian tokens reasonably well without having retrained the Transformer layers. Therefore, the English Transformer layers are at least partially language-independent and our relearning method automatically aligns lexical embeddings to the embedding space of the English model. However, if we retrain the med lexical layer for Italian and Dutch with the same method, test data perplexities are 81.2 and 185.0. These unsatisfactory med perplexities could be due to stopping training too early or to arriving at a suboptimal local optimum. Training for a longer time or trying different random initialisations defeats the purpose of minimising computational requirements. A more efficient method that uses the already learned sml embeddings is described in Section 5.

Vocabulary alignment The lexical embeddings of both the original English tokens as well as the relearned Italian and Dutch lexical embeddings can be considered to inhabit the same embedding space because the lexical embeddings of all three languages are tuned to minimise loss with the exact same Transformer layers. Therefore, tokens with

\begin{tabular}{l|ll}
\hline English & Italian & Dutch \\
\hline while & mentre & terwijl \\
genes & geni & genen \\
clothes & vestiti & kleren \\
musicians & composi[...] & artiesten \\
permitted & ammessa & toegelaten \\
Finally & infine & Eindelijk \\
satisfied & soddisfatto & tevreden \\
\hline Accuracy: & $85 \%$ & $89 \%$ \\
\hline
\end{tabular}

Table 1: Alignment of closest tokens in the lexical embeddings of $\mathrm{sml}_{\mathrm{rle}}$ for Italian and Dutch. Accuracy scores are based on a manual evaluation by the authors of 200 random aligned tokens. Semantically correct subword matches are included.

similar meaning in different languages should be close to each other if the lexical embeddings are properly trained. Table 1 shows the closest Italian and Dutch tokens of a random sample of English tokens. These alignments show that the optimal lexical embeddings for both Italian and Dutch are often literal translations of English tokens. Thanks to similarity of context-dependent structures like syntax in these three languages, the English model can be adapted to Italian and Dutch. Based on this small sample, Dutch to English alignment seems to be slightly more accurate than Italian to English, but a more thorough study would be required to evaluate the actual relation between genetic similarity and alignment potential through this method.

Text generation Table 2 shows some examples of unconditioned text generation of the English sml with relearned lexical embeddings for Italian and Dutch. These examples show that the model can generate proper Italian and Dutch sentences, although it sometimes uses English word order where the correct word order differs in Dutch, or ignores grammatical gender agreement in Italian defaulting to the singular masculine, or doesn't always produce correctly Italian prepositional articles ("di la", en: of the vs "della", en: of-the). Phrases in italics in Table 2 highlight such mistakes.

The literal English translations, however, show that the models can generate proper Italian and Dutch grammar that differs from English. Italian and Dutch lexical embeddings are not only aligned with equivalent English tokens, but unexpected correct syntax shows that the grammatical functions of words have also been adapted. For example, in Ital- 


\begin{tabular}{l|l}
\hline Italian & Literal English translation \\
\hline $\begin{array}{l}\text { La prima parte del film venne distribuito in Giappone con } \\
\text { l'aggiunta della colonna sonora. }\end{array}$ & $\begin{array}{l}\text { The first part of the film was distributed in Japan with the } \\
\text { addition of the soundtrack. }\end{array}$ \\
\hline $\begin{array}{l}\text { L'unico motivo di la mia insoddisfazione fu il fatto che } \\
\text { l'inizio della sua attività [...] }\end{array}$ & $\begin{array}{l}\text { The only reason of the my unsatisfaction was the fact that the } \\
\text { beginning of-the his/her activity [...] }\end{array}$ \\
\hline Il suo nome deriva da un vocabolo arabo. & The his/her name derives from a word Arabic. \\
\hline Dutch & Literal English translation \\
\hline $\begin{array}{l}\text { In een artikel in de Journal of Economicologie (1998), The } \\
\text { New York Times schrijft: }\end{array}$ & $\begin{array}{l}\text { In an article in the Journal of Economicology (1998), The } \\
\text { New York Times writes: }\end{array}$ \\
\hline $\begin{array}{l}\text { Ik kan me niet voorstellen dat mensen van mijn generatie } \\
\text { zijn zo boos op mij te wachten. }\end{array}$ & $\begin{array}{l}\text { I can me not imagine that people of my generation are so } \\
\text { mad at me to wait. }\end{array}$ \\
\hline Ik heb niets gedaan om mijn moeder te helpen. & I have nothing done to my mother to help. \\
\hline
\end{tabular}

Table 2: A selection of generated sentences by the sml model with Italian and Dutch lexical embeddings. Phrases in italics are ungrammatical in the target language.

ian the noun-adjective order is opposite to English and realised correctly; also, the use of the definite article in front of a possessive pronoun is correctly introduced, while ungrammatical in English.

This shows that the relatively low-dimensional context-independent lexical embeddings in GPT-2 contain syntactic features of the tokens in addition to semantics, and confirms previous findings of high information density in the lexical layer of language models (de Vries et al., 2020). Therefore, language adaptation can be to some extent effective by adapting the lexical embedding layer without retraining Transformer layers at all.

\section{Scaling up Complexity}

Replacing the original lexical embeddings with lexical embeddings from a different target language seems an effective way to initialise full model transfer to that target language. However, relearning the lexical embeddings of a new vocabulary requires full forward and backward propagation through the whole model. Therefore, this becomes an increasingly more expensive task for larger model sizes. When multiple model sizes need to be transferred to a new language, the lexical embeddings do not need to be retrained from scratch. Instead, vocabulary alignment between the source and target languages for the smaller model could be used to initialise the embeddings for a larger model.

After relearning the lexical embeddings of the sml model for Italian and Dutch, we observed that tokens with similar meaning in different languages are close to each other in the embedding space. This alignment effect should also be present in properly trained lexical embeddings of larger models. Given that we have at our disposal known embeddings for all 50K English tokens for every model size, we can use these data points to transform model size sml to larger model size med.

Regardless of architecture, embeddings are only considered to be alignable if they are trained under identical conditions with the same type and amount of data (Levy et al., 2015; Ruder et al., 2019). Our goal differs from previous alignment efforts since instead of aligning languages, we align separately trained embeddings for different model sizes, trained on the same data with identical and fully parallel vocabularies in English. The embeddings differ in dimensionality (768d for $\mathrm{sml}$, $1024 \mathrm{~d}$ for med) and the different model sizes may influence the amount and density of information in the lexical embeddings.

\subsection{Transformation methods}

The $50 \mathrm{~K}$ parallel English tokens can be used to find an optimal transformation between lexical embeddings of different model sizes. The completeness of this mapping due to shared vocabularies between models eliminates the need to use complex solutions like refinement or bootstrapping the lexicon (Artetxe et al., 2018). We compare three simple supervised alignment methods for transformation from source space sml to target space med.

Regression (Istsq) A classic approach for mapping lexical embeddings is mean-squared-error minimising linear regression with the least-squares method (Ruder et al., 2019; Mikolov et al., 2013). This method learns a transformation matrix $\boldsymbol{W}$ that minimises the Euclidean distance between source and target embeddings. The optimal matrix is ap- 


\begin{tabular}{|c|c|c|c|c|c|}
\hline \multirow[b]{2}{*}{ Model } & \multicolumn{2}{|c|}{ Italian } & \multicolumn{3}{|c|}{ Dutch } \\
\hline & Int@1k & PPL & Int@1k & PPL & PPL (1 epoch) \\
\hline $\operatorname{med}_{r l e}(1$ epoch $)$ & 0.38 & - & 185.02 & - & - \\
\hline $\mathrm{sml}_{\mathrm{rle}} \stackrel{\text { proc }}{\longrightarrow}$ med & 0.61 & $8.12 \times 10^{12}$ & 0.61 & $5.02 \times 10^{12}$ & 52.69 \\
\hline $\mathrm{sml}_{\mathrm{rle}} \stackrel{\text { lstsq }}{\longrightarrow}$ med & 0.56 & 364.06 & 0.56 & 293.61 & 47.57 \\
\hline $\mathrm{sml}_{\mathrm{rle}} \stackrel{1-n n}{\longrightarrow} \operatorname{med}$ & 0.37 & $2,764.19$ & 0.36 & $1,101.59$ & 50.25 \\
\hline $\mathrm{sml}_{\mathrm{rle}} \stackrel{10-n n}{\longrightarrow}$ med & 0.37 & $20,715.80$ & 0.35 & $11,871.66$ & 56.88 \\
\hline
\end{tabular}

Table 3: Scores for different transformation methods. Int@1K are the average 1k nearest English neighbours intersection (int) fractions between sml and transformed med embeddings. PPL is the perplexity on the test sets for Italian and Dutch. PPL (1 epoch) indicates the perplexity after one epoch of training, which is low if the transformed embeddings were close to a good local optimum.

proximated with stochastic gradient descent, and therefore this is not an exact solution.

Orthogonal Procrustes (proc) More recent alignment approaches constrain the transformation $\boldsymbol{W}$ to be an orthogonal matrix (Ruder et al., 2019; Artetxe et al., 2016). This constraint enables using the exact solution for the orthogonal Procrustes problem (Xing et al., 2015). The exact solution only rotates and reflects data points to be as close as possible to the target space without any scaling or translation, preserving monolingual invariance in the source embeddings (Artetxe et al., 2016).

Weighted K-Nearest Neighbours (knn) Unlike typical alignment approaches, we have a complete set of parallel data points in the source and target spaces (English). The unknown target language tokens can be approximated by taking the $\boldsymbol{K}$ nearest English tokens in the source sml embedding space and using the distance-weighted sum of these tokens in the target med embedding space.

\subsection{Results after transformation}

Table 3 shows med embedding similarity with source sml embeddings and perplexities on test data with transformed embeddings, as well as the transformed embeddings with one additional epoch of training. Results are consistent for Italian and Dutch. Nearest English neighbours are best preserved with the Orthogonal Procrustes method. However, the perplexity scores are extremely high for this method. The perplexity scores with the different methods vary in complete orders of magnitude. Based on this, the least-squares regression method outperforms the other methods. After one epoch of additional training with transformation initialised embeddings, the lstsq method still outper-

\begin{tabular}{l|cr}
\hline Model & \multicolumn{2}{|c}{ PPL } \\
& ita & nld \\
\hline sml rle $_{\text {sml }}+$ finetuning & 44.19 & 48.85 \\
sml full & $\mathbf{4 2 . 4 5}$ & $\mathbf{3 9 . 5 9}$ \\
\hline medrle $_{\text {r finetuning }}$ & 42.51 & 44.68 \\
\hline GePpeTto (sml) & 106.84 & - \\
\hline
\end{tabular}

Table 4: Perplexities of the concatenated test data for the final models. The medrle model is in practice the $\mathrm{sml}_{\text {rle }} \stackrel{\text { lstsq }}{\longrightarrow}$ med model. * The $\mathrm{sml}_{\text {full }}$ model is trained for the equivalent amount of time as the $s m l_{r l e}$ + finetuning models, but with all layers unfrozen.

forms the other methods. It even outperforms the sml model with fully tuned lexical embeddings.

\section{Full model finetuning}

After obtaining lexical embeddings for Italian and Dutch to be plugged into the English GPT-2 models, the full models can be finetuned for the target language. The best performing lexical embeddings will be used to train the sml and med Italian and Dutch models. These are the lexical embeddings that are relearned from random initialisation for the sml model. For the med model, the lstsq transformed sml embeddings with additional training are used $\left(\mathrm{sml}_{\mathrm{rle}} \stackrel{\text { lstsq }}{\longrightarrow}\right.$ med $\left._{+\mathrm{rle}}\right)$.

The relearned lexical embeddings reduce the risk of information loss while the model is adjusting to a new language. Nevertheless, information can still be lost during training. For instance for the sml Dutch model, validation loss increases with a learning rate of $10^{-4}$, but this does not happen with a lower learning rate of $10^{-5}$. 


\section{Obtained models and evaluation}

For both Italian and Dutch, we evaluate three models: (i) the English sml model with relearned lexical embeddings; (ii) the sml model with additional finetuning to the target language; and (iii) the English med model with relearned lexical embeddings that were initialised by transforming sml embeddings with the least-squares method. For Italian, we also include the GPT- 2 small based GePpeTto model (De Mattei et al., 2020), which was trained from scratch. This inclusion offers the opportunity of a direct comparison between a GPT-2 model trained from scratch and those obtained with our transfer approach. We run both an automatic and a human-based evaluation. For the former, we compare perplexity scores on unseen test data in different genres. For the latter, we collect and compare judgements over generated and gold texts by native speakers of Italian and Dutch.

\subsection{Perplexity}

Table 4 shows perplexity scores on concatenated multi-genre test data based on a strided moving window perplexity calculation. ${ }^{3}$ Perplexities are calculated with Italian and Dutch vocabularies of $30 \mathrm{~K}$ tokens. These results show that perplexities are low when only relearning the lexical embeddings for both Italian and Dutch. Further finetuning of the sml model seems to have the greatest effect for the Dutch language. The med models with relearned lexical embeddings have lower perplexity than the equivalent sml models. This shows that language transferability based on the lexical layer is not restricted to small model sizes. Moreover, we see that our proposed method results in lower perplexity scores than regular full model finetuning of the English model. The overall perplexity scores of Italian are closer to each other than the Dutch perplexities. We also tested perplexities by the different genres that make up both the Italian and the Dutch datasets (see Figure 5 and Figure 6 for details), and observed that while perplexities vary greatly per genre, the model ranking per genre is consistent with the global scores.

\subsection{Human Judgements}

The perplexity scores give an indication on how well a language is represented by language models,

\footnotetext{
${ }^{3}$ Window sizes are 128 tokens and strides are 64 tokens except for GePpeTto. GePpeTto was trained with at most 100 tokens, so its window size is 100 with a 50 token stride.
}

\begin{tabular}{l|ccc}
\hline Model & Social & News & Legal \\
\hline sml $_{\text {rle }}$ & 134.64 & 67.14 & 16.95 \\
sml rle + finetuning & $\mathbf{1 1 8 . 1 9}$ & $\mathbf{5 5 . 6 3}$ & 15.36 \\
\hline med $_{\text {rle }}$ & 123.64 & 59.18 & $\mathbf{1 4 . 9 5}$ \\
\hline GePpeTto & 179.47 & 80.83 & 34.71 \\
\hline
\end{tabular}

Table 5: Perplexities for different genres within the Italian test data. Rankings are consistent with Table 4 except for the legal domain.

\begin{tabular}{l|ccc}
\hline Model & Proceedings & News & Legal \\
\hline $\mathrm{sml}_{\text {rle }}$ & 44.47 & 239.14 & 52.01 \\
$\mathrm{sml}_{\mathrm{rle}}+$ finetuning & $\mathbf{3 6 . 3 5}$ & $\mathbf{1 7 1 . 8 3}$ & $\mathbf{4 2 . 9 2}$ \\
\hline med $_{\mathrm{rle}}$ & 40.62 & 234.52 & 45.01 \\
\hline
\end{tabular}

Table 6: Perplexities for some SoNaR genres in Dutch. Models rankings are consistent across genres.

but this does not reliably tell how good the model is in a generative setting. For this, we resort to human judgements. Human assessments of generated texts are collected for the models that incorporate the crucial steps in our approach and achieve reasonable perplexity scores: the sml models with only relearned lexical embeddings, the finetuned sml models and the higher complexity med models with only relearned lexical embeddings based on transformed sml lexical embeddings.

Texts are assessed in isolation by means of a direct evaluation (Novikova et al., 2018). ${ }^{4}$ Subjects are presented with texts on the screen, and are asked whether the texts they see could have been written by a human. All subjects are pre-informed that some of the texts they will see are machine generated. Rather than discrete answers, we obtain continuous evaluations by offering the possibility of clicking anywhere on a bar whose extremes are "no" to the left and "yes" to the right.

The evaluation interface is made with PsychoPy3 (Peirce et al., 2019) and hosted with Pavlovia ${ }^{5}$.

Italian models were evaluated by 24 participants $(9 \mathrm{M}, 15 \mathrm{~F})$ with ages ranging from 26 to 63 with a median age of 46 . The Dutch models were evaluated by 15 participants $(11 \mathrm{M}, 4 \mathrm{~F})$ with ages ranging from 23 to 36 with a median age of 27 .

The three final models are evaluated for both languages; for Italian, we also add GePpeTto (De Mattei et al., 2020). Human written gold sentences

\footnotetext{
${ }^{4} \mathrm{~A}$ direct evaluation is opposed to a comparative one, usually involving a ranking task (Novikova et al., 2018; De Mattei et al., 2020); this is left to future work.

${ }^{5}$ https: / / pavlovia.org
} 


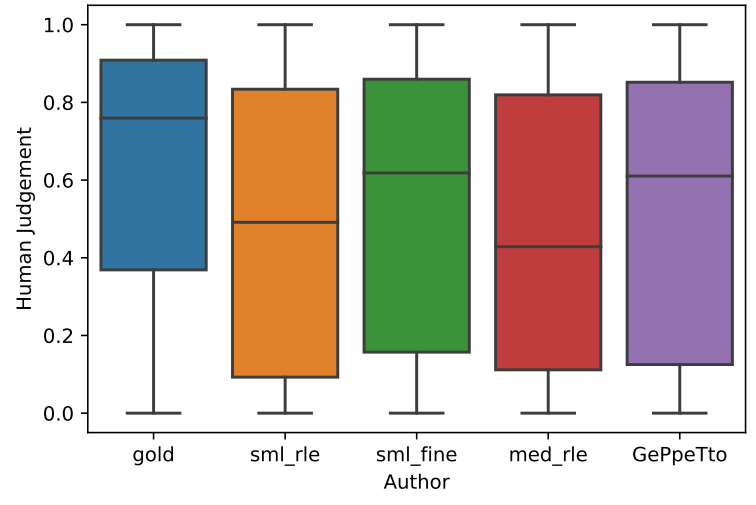

(a) Human judgement scores for Italian texts.

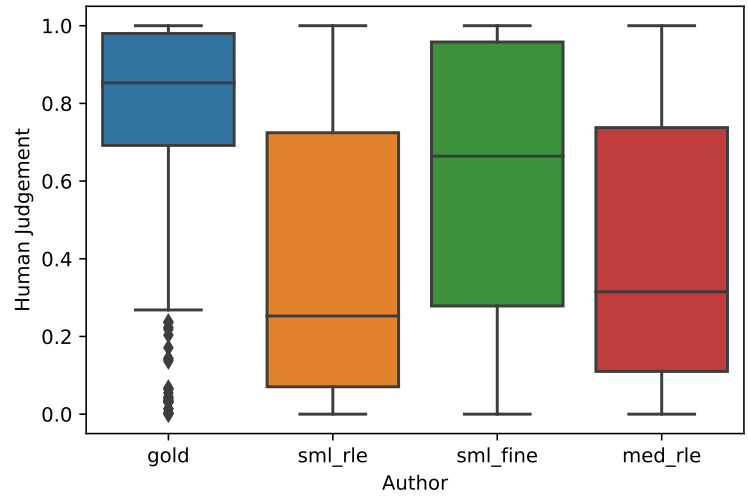

(b) Human judgement scores for Dutch texts.

Figure 1: Human judgement scores based on a continuous scale. Most judgements were close to 0 or 1.

were sampled from the test data as an additional condition. For each of these 5 Italian and 4 Dutch conditions, 100 sentences are evaluated. Each participant has evaluated 50 to 150 sentences and each sentence is evaluated by 3 to 5 participants. As a result, we obtain 1950 evaluations for 500 Italian texts and 1550 evaluations for 400 Dutch texts.

All artificial sentences are randomly generated without conditioning and with beam search (5 beams, with top 50 tokens or a summed probability of at least 90\%), and a temperature of 3.0. Setting the temperature value $>1$ means decreasing the sampling probability of likely tokens, and therefore increases variation between generated samples.

Longer sentences have a higher chance to contain mistakes, so a model that generates longer sentences may have a disadvantage. However, explicitly controlling sentence length is not possible nor desired since sentence length may also be an indication of model quality. For both languages the randomly sampled gold sentences have more long sentences than the models, but the sml model with finetuning also sometimes generates longer sentences. We filter out sentences longer than 30 tokens to decrease sentence length effects on judgements. The remaining Italian sentences have median lengths of 18 or 22 words and the Dutch ones 16 or 17 words for the different conditions.

Figure 1 shows the distributions of human judgements per condition. Variance seems to be high due to the non-normally distributed scores as relatively many scores are close to zero, half or one. The model differences appear stronger for Dutch than Italian, but for both languages the subjects have given high scores to gold sentences. This is expected and indicates that the participants are able to correctly judge real human texts. Of the three trained models, the small model with additional finetuning achieves the highest scores.

For the Italian model comparison we use a linear mixed-effects model but with only author as fixed effect and random intercepts for participants and sentences. There is no significant effect for sentence length. The judgements on gold texts are significantly higher than all model judgements $(p<0.005)$ except for $\mathrm{sml}_{\mathrm{fine}}$. However, $s m l_{\text {fine }}$ is not significantly better than GePpeTto nor the $\mathrm{sml}_{\mathrm{rle}}$ and med $\mathrm{d}_{\mathrm{rle}}$ models $(p>0.05)$.

For Dutch we use a linear mixed-effects model with fixed effects for author and sentence length (in number of words) and random intercepts for participants and sentences. Sentence length has a significant negative effect $(p<0.001)$. All artificial authors score significantly lower than gold $(p<0.001)$. As for Italian, the $s m I_{\text {fine }}$ model appears the best model, but in this case the judgement scores are significantly higher than for the other two models $(p<0.001)$. The $\mathrm{sml}_{\mathrm{rle}}$ and medrle models do not differ significantly from each other.

The human judgements show consistent results across the languages, but differences between Dutch judgements are stronger than for Italian. This seems to mirror the smaller perplexity differences for Italian than for Dutch. Whether demographic or cultural differences also play a role in this difference will need to be further investigated.

In sum, we see that the English GPT-2 models with relearned lexical embeddings are recognisable as artificial, whereas this problem is attenuated after additional finetuning. The sml model with additional finetuning performs at least as well as the GePpeTto model that was trained from scratch. 


\section{Conclusion}

We have described methods to adapt GPT-2 to genetically related languages and to increase model complexity. Retraining lexical embeddings forces the model to learn representations that are aligned between English and the target language. GPT-2 is able to generate realistic text in another language, but human judgements reveal that additional finetuning of the full model is needed to generate realistic sentences more consistently. Relearned lexical embeddings show signs of syntactic adaptation to the new language, though not fully consistently.

Dutch is genetically closer to English than Italian, but our results do not prove that this method works better for Dutch. Future research on the relation between degrees and types of language similarity and transferability of models will enable more effective monolingual transfer, and possibly training better multilingual models by selecting optimal clusters of languages. This kind of work offers a privileged perspective into the information learned by generative language models and provides empirical ground for linguistic typology research (e.g., uncovering which linguistic aspects are more universal, and which more language-specific).

Relearning lexical embeddings using our method can still be considered an expensive solution, but training costs decrease when a smaller embedding space is scaled up to the embedding space of a larger model. In other words, approximating a good initialisation of the embedding weights decreases training time. This method also enables adaptation of (extra) large GPT-2 models to other languages.

If you can borrow pre-trained weights, why retrain models from scratch? In the right column we summarise the steps for the shortest path to train your own GPT-2 for another language.

\section{Acknowledgments}

We gratefully acknowledge the support of the Dutch Research Council (NWO Aspasia grant for M. Nissim) and the financial support of the Center for Groningen Language and Culture (CGTC). Additionally, we would like to thank Lorenzo De Mattei for sharing the Italian data with us. We would also like to thank the Center for Information Technology of the University of Groningen for providing access to the Peregrine high performance computing cluster. Finally, we thank the anonymous reviewers for their insightful feedback. Any mistakes remain our own.

\section{Impact Statement}

This work aims to minimize the environmental impact of training large neural language models by adapting existing models and by using smart initialisation of model weights. However, experiments in this paper still require the use of GPUs for extended periods of time which has environmental impact. Our final models are published and all models that automatically generates natural text could unfortunately be used maliciously. While we cannot fully prevent such uses once our models are made public, we do hope that writing about risks explicitly and also raising awareness of this possibility in the general public are ways to contain the effects of potential harmful uses. We are open to any discussion and suggestions to minimise such risks.

\section{Create your own GPT-2 model}

This paper describes several steps that are taken to transfer GPT-2 to a different language. The recommended shortest path to replicate this for another language is to follow these steps:

Vocabulary Create a new BPE vocabulary for your target language. The optimal size for your vocabulary depends on your language, so select the size by stepwise increments until the number of tokens per sentence slows to decrease.

Start small Re-initialise the lexical embeddings of the small GPT-2 model for your vocabulary size and only retrain the lexical embeddings.

Increase model size If you want to train a larger model size, fit a least-squares regression model to the English lexical embeddings in the small and larger model size and use the fitted model to transform your newly trained lexical embeddings to a larger model size.

Optimise your embeddings Do additional lexical embedding training in the target model size. Transformed embeddings are a good initialisation, but they are not perfect.

Finetune Unfreeze the full target model and do some finetuning to make sure that syntax differences are learned by the new model. Use a low learning rate like $10^{-5}$. 


\section{References}

Mikel Artetxe, Gorka Labaka, and Eneko Agirre. 2016. Learning principled bilingual mappings of word embeddings while preserving monolingual invariance. In Proceedings of the 2016 Conference on Empirical Methods in Natural Language Processing, pages 2289-2294, Austin, Texas. Association for Computational Linguistics.

Mikel Artetxe, Gorka Labaka, Eneko Agirre, and Kyunghyun Cho. 2018. Unsupervised Neural Machine Translation. arXiv:1710.11041 [cs]. ArXiv: 1710.11041 .

Mikel Artetxe, Sebastian Ruder, and Dani Yogatama. 2020. On the Cross-lingual Transferability of Monolingual Representations. In Proceedings of the 58th Annual Meeting of the Association for Computational Linguistics, pages 4623-4637, Online. Association for Computational Linguistics.

Marco Baroni, Silvia Bernardini, Adriano Ferraresi, and Eros Zanchetta. 2009. The WaCky wide web: a collection of very large linguistically processed webcrawled corpora. Language Resources and Evaluation, 43(3):209-226.

Alexis Conneau, Kartikay Khandelwal, Naman Goyal, Vishrav Chaudhary, Guillaume Wenzek, Francisco Guzmán, Edouard Grave, Myle Ott, Luke Zettlemoyer, and Veselin Stoyanov. 2020. Unsupervised Cross-lingual Representation Learning at Scale. In Proceedings of the 58th Annual Meeting of the Association for Computational Linguistics, pages 8440 8451, Online. Association for Computational Linguistics.

Alexis Conneau, Guillaume Lample, Marc'Aurelio Ranzato, Ludovic Denoyer, and Hervé Jégou. 2018. Word Translation Without Parallel Data. arXiv:1710.04087 [cs]. ArXiv: 1710.04087.

Lorenzo De Mattei, Michele Cafagna, Felice Dell'Orletta, Malvina Nissim, and Marco Guerini. 2020. GePpeTto Carves Italian into a Language Model. arXiv:2004.14253 [cs].

Pieter Delobelle, Thomas Winters, and Bettina Berendt. 2020. RobBERT: a Dutch RoBERTa-based Language Model. arXiv:2001.06286 [cs].

David M. Eberhard, Gary F. Simons, and Charles D. Fennig. 2020. Ethnologue: Languages of the World. Twenty-third edition. SIL International.

W.A. Falcon. 2019. PyTorch Lightning.

Goran Glavaš, Robert Litschko, Sebastian Ruder, and Ivan Vulić. 2019. How to (Properly) Evaluate CrossLingual Word Embeddings: On Strong Baselines, Comparative Analyses, and Some Misconceptions. In Proceedings of the 57th Annual Meeting of the Association for Computational Linguistics, pages 710 721, Florence, Italy. Association for Computational Linguistics.
Armand Joulin, Piotr Bojanowski, Tomas Mikolov, Hervé Jégou, and Edouard Grave. 2018. Loss in Translation: Learning Bilingual Word Mapping with a Retrieval Criterion. In Proceedings of the 2018 Conference on Empirical Methods in Natural Language Processing, pages 2979-2984, Brussels, Belgium. Association for Computational Linguistics.

Armand Joulin, Edouard Grave, Piotr Bojanowski, and Tomas Mikolov. 2017. Bag of Tricks for Efficient Text Classification. In Proceedings of the 15th Conference of the European Chapter of the Association for Computational Linguistics: Volume 2, Short Papers, pages 427-431, Valencia, Spain. Association for Computational Linguistics.

Yunsu Kim, Yingbo Gao, and Hermann Ney. 2019. Effective Cross-lingual Transfer of Neural Machine Translation Models without Shared Vocabularies. In Proceedings of the 57th Annual Meeting of the Association for Computational Linguistics, pages 12461257, Florence, Italy. Association for Computational Linguistics.

Diederik P. Kingma and Jimmy Ba. 2017. Adam: A Method for Stochastic Optimization. In arXiv:1412.6980 [cs].

Tom Kocmi and Ondřej Bojar. 2018. Trivial Transfer Learning for Low-Resource Neural Machine Translation. In Proceedings of the Third Conference on Machine Translation: Research Papers, pages 244 252, Brussels, Belgium. Association for Computational Linguistics.

Omer Levy, Yoav Goldberg, and Ido Dagan. 2015. Improving Distributional Similarity with Lessons Learned from Word Embeddings. Transactions of the Association for Computational Linguistics, 3:211-225.

Yu-Hsiang Lin, Chian-Yu Chen, Jean Lee, Zirui Li, Yuyan Zhang, Mengzhou Xia, Shruti Rijhwani, Junxian He, Zhisong Zhang, Xuezhe Ma, Antonios Anastasopoulos, Patrick Littell, and Graham Neubig. 2019. Choosing Transfer Languages for CrossLingual Learning. In Proceedings of the 57th Annual Meeting of the Association for Computational Linguistics, pages 3125-3135, Florence, Italy. Association for Computational Linguistics.

Paulius Micikevicius, Sharan Narang, Jonah Alben, Gregory Diamos, Erich Elsen, David Garcia, Boris Ginsburg, Michael Houston, Oleksii Kuchaiev, Ganesh Venkatesh, and Hao Wu. 2018. Mixed Precision Training. arXiv:1710.03740 [cs, stat]. ArXiv: 1710.03740 .

Tomas Mikolov, Quoc V. Le, and Ilya Sutskever. 2013. Exploiting Similarities among Languages for Machine Translation. arXiv:1309.4168 [cs].

Tasnim Mohiuddin, M Saiful Bari, and Shafiq Joty. 2020. LNMap: Departures from Isomorphic Assumption in Bilingual Lexicon Induction Through 
Non-Linear Mapping in Latent Space. In Proceed ings of the 2020 Conference on Empirical Methods in Natural Language Processing (EMNLP), pages 2712-2723, Online. Association for Computational Linguistics.

Toan Q. Nguyen and David Chiang. 2017. Transfer Learning across Low-Resource, Related Languages for Neural Machine Translation. In Proceedings of the Eighth International Joint Conference on Natural Language Processing (Volume 2: Short Papers), pages 296-301, Taipei, Taiwan. Asian Federation of Natural Language Processing.

Jekaterina Novikova, Ondřej Dušek, and Verena Rieser. 2018. RankME: Reliable Human Ratings for Natural Language Generation. In Proceedings of the 2018 Conference of the North American Chapter of the Association for Computational Linguistics: $\mathrm{Hu}$ man Language Technologies, Volume 2 (Short Papers), pages 72-78, New Orleans, Louisiana. Association for Computational Linguistics.

Debora Nozza, Federico Bianchi, and Dirk Hovy. 2020. What the [MASK]? Making Sense of LanguageSpecific BERT Models. arXiv:2003.02912 [cs].

Nelleke Oostdijk, Martin Reynaert, Véronique Hoste, and Ineke Schuurman. 2013. The Construction of a 500-Million-Word Reference Corpus of Contemporary Written Dutch. In Peter Spyns and Jan Odijk, editors, Essential Speech and Language Technology for Dutch: Results by the STEVIN programme, Theory and Applications of Natural Language Processing, pages 219-247. Springer, Berlin, Heidelberg.

Ordelman, Roeland J.F., de Jong, Franciska M.G., van Hessen, Adrianus J., and Hondorp, G.H.W. 2007. TwNC: a Multifaceted Dutch News Corpus. ELRA Newsletter, 12(3-4).

Adam Paszke, Sam Gross, Francisco Massa, Adam Lerer, James Bradbury, Gregory Chanan, Trevor Killeen, Zeming Lin, Natalia Gimelshein, Luca Antiga, Alban Desmaison, Andreas Kopf, Edward Yang, Zachary DeVito, Martin Raison, Alykhan Tejani, Sasank Chilamkurthy, Benoit Steiner, Lu Fang, Junjie Bai, and Soumith Chintala. 2019. PyTorch: An Imperative Style, High-Performance Deep Learning Library. In H. Wallach, H. Larochelle, A. Beygelzimer, F. d \textquotesingle Alché-Buc, E. Fox, and R. Garnett, editors, Advances in Neural Information Processing Systems 32, pages 80268037. Curran Associates, Inc.

Jonathan Peirce, Jeremy R. Gray, Sol Simpson, Michael MacAskill, Richard Höchenberger, Hiroyuki Sogo, Erik Kastman, and Jonas Kristoffer Lindeløv. 2019. PsychoPy2: Experiments in behavior made easy. Behavior Research Methods, 51(1):195-203.

Alec Radford, Jeffrey Wu, Rewon Child, David Luan, Dario Amodei, and Ilya Sutskever. 2019. Language Models are Unsupervised Multitask Learners.
Sebastian Ruder, Ivan Vulić, and Anders Søgaard. 2019. A Survey of Cross-lingual Word Embedding Models. Journal of Artificial Intelligence Research, 65:569-631.

Leslie N. Smith. 2017. Cyclical Learning Rates for Training Neural Networks. In 2017 IEEE Winter Conference on Applications of Computer Vision $(W A C V)$, pages 464-472.

Emma Strubell, Ananya Ganesh, and Andrew McCallum. 2019. Energy and Policy Considerations for Deep Learning in NLP. In Proceedings of the 57th Annual Meeting of the Association for Computational Linguistics, pages 3645-3650, Florence, Italy. Association for Computational Linguistics.

Anders Søgaard, Sebastian Ruder, and Ivan Vulić. 2018. On the Limitations of Unsupervised Bilingual Dictionary Induction. In Proceedings of the 56th Annual Meeting of the Association for Computational Linguistics (Volume 1: Long Papers), pages 778788, Melbourne, Australia. Association for Computational Linguistics.

Wietse de Vries, Andreas van Cranenburgh, and Malvina Nissim. 2020. What's so special about BERT's layers? A closer look at the NLP pipeline in monolingual and multilingual models. In Findings of the Association for Computational Linguistics: EMNLP 2020, pages 4339-4350, Online. Association for Computational Linguistics.

Wietse de Vries, Andreas van Cranenburgh, Arianna Bisazza, Tommaso Caselli, Gertjan van Noord, and Malvina Nissim. 2019. BERTje: A Dutch BERT Model. arXiv:1912.09582 [cs].

Thomas Wolf, Lysandre Debut, Victor Sanh, Julien Chaumond, Clement Delangue, Anthony Moi, Pierric Cistac, Tim Rault, Rémi Louf, Morgan Funtowicz, and Jamie Brew. 2020. HuggingFace's Transformers: State-of-the-art Natural Language Processing. arXiv:1910.03771 [cs].

Shijie Wu and Mark Dredze. 2020. Are All Languages Created Equal in Multilingual BERT? arXiv:2005.09093 [cs]. ArXiv: 2005.09093.

Chao Xing, Dong Wang, Chao Liu, and Yiye Lin. 2015. Normalized Word Embedding and Orthogonal Transform for Bilingual Word Translation. In Proceedings of the 2015 Conference of the North American Chapter of the Association for Computational Linguistics: Human Language Technologies, pages 1006-1011, Denver, Colorado. Association for Computational Linguistics.

Barret Zoph, Deniz Yuret, Jonathan May, and Kevin Knight. 2016. Transfer Learning for Low-Resource Neural Machine Translation. In Proceedings of the 2016 Conference on Empirical Methods in Natural Language Processing, pages 1568-1575, Austin, Texas. Association for Computational Linguistics. 